\title{
CHROMATIC NUMBER OF CARTESIAN SUM OF TWO GRAPHS
}

\section{KUNG-WEI YANG}

In this note, we will consider the class of all finite undirected graphs with simple edges and no loops [1]. Let $G, G_{1}, G_{2}$ denote graphs. Let $o(G)=$ the number of vertices in $G, \beta(G)=$ the independence number of $G, \mathrm{~K}(G)=$ the chromatic number of $G, G_{1} \oplus G_{2}=$ the Cartesian sum of $G_{1}$ and $G_{2}[1]$. We shall prove the following

Theorem. $o\left(G_{1}\right) o\left(G_{2}\right) / \beta\left(G_{1}\right) \beta\left(G_{2}\right) \leqq \mathrm{K}\left(G_{1} \oplus G_{2}\right) \leqq \mathrm{K}\left(G_{1}\right) \mathrm{K}\left(G_{2}\right)$.

Moreover, an example is given to show that the inequality $\mathrm{K}\left(G_{1} \oplus G_{2}\right)<\mathrm{K}\left(G_{1}\right) \mathrm{K}\left(G_{2}\right)$ in fact occurs.

The proof is based on the following two lemmas.

Lemma 1. $\beta\left(G_{1} \oplus G_{2}\right)=\beta\left(G_{1}\right) \beta\left(G_{2}\right)$.

Proof. If $G$ is a graph, we denote by $V(G)$ the set of vertices of $G$ and by $E(G)$ the set of edges of $G$. We say that a subset $S \subset V(G)$ is independent if for any $a, b$ in $S,(a, b) \notin E(G)$. If $S$ is a set, we denote by $|S|$ the number of elements in $S$. Now, let $S_{i} \subset V\left(G_{i}\right)(i=1,2)$ be independent sets such that $\left|S_{i}\right|=\beta\left(G_{i}\right)$. If $S=\left\{a_{1} \oplus a_{2}: a_{1} \in S_{1}\right.$ and $\left.a_{2} \in S_{2}\right\}$, then $S$ is an independent set of $G_{1} \oplus G_{2}$. Therefore, $\beta\left(G_{1} \oplus G_{2}\right)$ $\geqq|S|=\beta\left(G_{1}\right) \beta\left(G_{2}\right)$.

Suppose $T \subset V\left(G_{1} \oplus G_{2}\right)$ is an independent set such that $|T|$ $=\beta\left(G_{1} \oplus G_{2}\right)$. For $a \in V\left(G_{1}\right)$, let $T(a)=\left\{b \in V\left(G_{2}\right): a \oplus b \in T\right\}$. Then for each $a \in V\left(G_{1}\right), T(a)$ is an independent set of $G_{2}$. Therefore, for each $a \in V\left(G_{1}\right),|T(a)| \leqq \beta\left(G_{2}\right)$. Clearly, $|T|=\sum_{a \in V\left(G_{1}\right)}|T(a)|$. But $|T(a)|=0$ except for those $a$ in an independent set of $G_{1}$. Hence $\beta\left(G_{1} \oplus G_{2}\right)=|T| \leqq \beta\left(G_{1}\right) \beta\left(G_{2}\right)$. This shows that $\beta\left(G_{1} \oplus G_{2}\right)=\beta\left(G_{1}\right) \beta\left(G_{2}\right)$.

LEMmA 2. $\mathrm{K}\left(G_{1}\right) \mathrm{K}\left(G_{2}\right) \geqq \mathrm{K}\left(G_{1} \oplus G_{2}\right)$.

Proof. Let $G, H$ be graphs. A homomorphism $f: G \rightarrow H$ is a map $f: V(G) \rightarrow V(H)$ such that if $(a, b) \in E(G)$, then $(f(a), f(b)) \in E(H)$. We say that a homomorphism $f: G \rightarrow H$ is surjective if $f: V(G) \rightarrow V(H)$ is surjective. If we denote by $K_{n}$ the complete graph of $n$ vertices, then the chromatic number of a graph $G$ can be defined as the smallest integer $n$ such that there exists a surjective homomorphism $f: G \rightarrow K_{n}$. Now let $m=\mathrm{K}\left(G_{1}\right)$ and $n=\mathrm{K}\left(G_{2}\right)$ and let $f_{1}: G_{1} \rightarrow K_{m}$ and $f_{2}: G_{2} \rightarrow K_{n}$ be surjective homomorphisms. If we define

Received by the editors February 14, 1967. 
by

$$
f_{1} \oplus f_{2}: G_{1} \oplus G_{2} \rightarrow K_{m} \oplus K_{n}
$$

$$
f_{1} \oplus f_{2}\left(a_{1} \oplus a_{2}\right)=f_{1}\left(a_{1}\right) \oplus f_{2}\left(a_{2}\right),
$$

then $f_{1} \oplus f_{2}$ is obviously a surjective homomorphism. Since $K_{m} \oplus K_{n}$ $\cong K_{m n}$, we obtain $\mathrm{K}\left(G_{1} \oplus G_{2}\right) \leqq \mathrm{K}\left(G_{1}\right) \cdot \mathrm{K}\left(G_{2}\right)$.

Proof of the Theorem. We only need to show that

$$
\left(o\left(G_{1}\right) o\left(G_{2}\right) / \beta\left(G_{1}\right) \beta\left(G_{2}\right)\right) \leqq \mathrm{K}\left(G_{1} \oplus G_{2}\right) .
$$

By $[1$, Theorem 14.1.1, p. 225], we have

$$
\left(o\left(G_{1} \oplus G_{2}\right) / \beta\left(G_{1} \oplus G_{2}\right)\right) \leqq \mathrm{K}\left(G_{1} \oplus G_{2}\right) .
$$

By Lemma 1, $\beta\left(G_{1} \oplus G_{2}\right)=\beta\left(G_{1}\right) \beta\left(G_{2}\right)$. It is obvious that $o\left(G_{1} \oplus G_{2}\right)$ $=o\left(G_{1}\right) o\left(G_{2}\right)$. Therefore, $\left(o\left(G_{1}\right) o\left(G_{2}\right) / \beta\left(G_{1}\right) \beta\left(G_{2}\right)\right) \leqq \mathrm{K}\left(G_{1} \oplus G_{2}\right)$.

Finally, we present an example to show that the inequality $\mathrm{K}\left(G_{1} \oplus G_{2}\right)<\mathrm{K}\left(G_{1}\right) \mathrm{K}\left(G_{2}\right)$ actually occurs.

Let $G_{1}$ be the graph with

$$
V\left(G_{1}\right)=\{1,2,3,4,5\}, \quad E\left(G_{1}\right)=\{(1,2),(2,3),(3,4),(4,5),(5,1)\} .
$$

Let $G_{2}$ be the same graph with different labeling of the vertices, say,

$$
\begin{aligned}
& V\left(G_{2}\right)=\left\{1^{\prime}, 2^{\prime}, 3^{\prime}, 4^{\prime}, 5^{\prime}\right\}, \\
& E\left(G_{2}\right)=\left\{\left(1^{\prime}, 2^{\prime}\right),\left(2^{\prime}, 3^{\prime}\right),\left(3^{\prime}, 4^{\prime}\right),\left(4^{\prime}, 5^{\prime}\right),\left(5^{\prime}, 1^{\prime}\right)\right\} .
\end{aligned}
$$

Clearly, $\mathrm{K}\left(G_{1}\right)=\mathrm{K}\left(G_{2}\right)=3$. We now define a surjective homomorphism $f: G_{1} \oplus G_{2} \rightarrow K_{8}$, where $V\left(K_{8}\right)=\left\{k_{1}, k_{2}, \cdots, k_{8}\right\}$, by

$$
\begin{aligned}
& f^{-1}\left[k_{1}\right]=\left\{1 \oplus 1^{\prime}, 1 \oplus 3^{\prime}, 3 \oplus 1^{\prime}, 3 \oplus 3^{\prime}\right\}, \\
& f^{-1}\left[k_{2}\right]=\left\{1 \oplus 2^{\prime}, 1 \oplus 4^{\prime}, 3 \oplus 2^{\prime}, 3 \oplus 4^{\prime}\right\}, \\
& f^{-1}\left[k_{3}\right]=\left\{2 \oplus 1^{\prime}, 2 \oplus 3^{\prime}, 4 \oplus 1^{\prime}, 4 \oplus 3^{\prime}\right\}, \\
& f^{-1}\left[k_{4}\right]=\left\{2 \oplus 2^{\prime}, 2 \oplus 4^{\prime}, 4 \oplus 2^{\prime}, 4 \oplus 4^{\prime}\right\}, \\
& f^{-1}\left[k_{5}\right]=\left\{3 \oplus 5^{\prime}, 5 \oplus 3^{\prime}, 5 \oplus 5^{\prime}\right\}, \\
& f^{-1}\left[k_{6}\right]=\left\{2 \oplus 5^{\prime}, 5 \oplus 2^{\prime}\right\}, \\
& f^{-1}\left[k_{7}\right]=\left\{1 \oplus 5^{\prime}, 4 \oplus 5^{\prime}\right\}, \\
& f^{-1}\left[k_{8}\right]=\left\{5 \oplus 1^{\prime}, 5 \oplus 4^{\prime}\right\} .
\end{aligned}
$$

The example shows that $\mathrm{K}\left(G_{1} \oplus G_{2}\right) \leqq 8<9=\mathrm{K}\left(G_{1}\right) \mathrm{K}\left(G_{2}\right)$.

\section{BiBLIOGRAPHY}

1. O. Ore, Theory of graphs, Amer. Math. Soc. Colloq. Publ., Vol. 38, Amer. Math. Soc., Providence, R. I., 1962.

Western Michigan University 